\title{
LOW-LUMINOSITY STARS: PAST AND FUTURE
}

\author{
I. N. REID \\ 105-24, California Institute of Technology \\ Pasadena, CA 91125
}

The expression "low luminosity stars" is a descriptive category which spans a wide range of objects, from the oldest stellar remnants in the Galaxy (Wood) through failed stars (Jones, Gould) to the enigmatic MACHOs, discernible not in themselves but only through their effects on others (Bennet). All of these have attracted considerable attention in recent years, and significant progress has been achieved in each case, even if our understanding has failed to keep up with observations. However, it is in the area of brown dwarfs where the most dramatic results have been obtained. The existence of such objects has been predicted theoretically for well over thirty years, but predictions can fail. Thus the discovery of GI229B (Nakajima et al, 1995), an unambiguous substellar-mass object, followed by the detection of lithium in Teide 1 and Calar 3 in the Pleiades (Rebolo et al, 1996) mark a turning point in studies of brown dwarfs. The issue now is not whether they exist, but what are their properties as a class. With that in mind, a number of obvious questions arise.

First, what is the most effective method of finding brown dwarfs? Both brown dwarfs and VLM stars have effective temperatures below $2500 \mathrm{~K}$. Hence, many surveys concentrated on searching for objects which were very red at near-infrared wavelengths, and the blue JHK colours exhibited by Gl 229B came as something of a surprise. This should not have been the case, of course, since Tsuji (1964) predicted the presence of methane in such cool atmospheres well before the term 'brown dwarf' was invented. Current theory, as summarised in these sessions by Tsuji and Burrows, predicts that, as one moves to lower temperatures, grain formation initially drives (J-K) redward, before grains settle below $\tau \sim 1$ and $\mathrm{CH}_{4}$ absorption sets in. Throughout, however, the optical-to-IR colours, straddling the peak in the emergent flux distribution, become progressively redder (Reid, 1994). Those colours therefore offer the most effective means of identifying both GD 165B-like and Gl 229B-like sources - an argument reinforced by the first results from the IJK DENIS survey (Delfosse et al, 1997).

Second, given that one can construct a statistically well-defined sample of VLM dwarfs and brown dwarfs, how does one derive the mass function, $\Psi(M)$, from the luminosity function, $\Phi(\mathrm{M})$ ? Clearly, the mass-luminosity relation is the key. As pointed out originally by D'Antona and Mazzitelli (1983), features in $\Phi(\mathrm{M})$ may stem from features in $\frac{d M}{d L}$, not $\Psi(M)$, but, despite the heroic efforts summarised here by Latham, the mass-luminosity relation remains poorly-constrained observationally below $1 \mathrm{M}_{\odot}$. It is particularly disconcerting that the one eclipsing system with $M<0.5 M_{\odot}$, CM Dra, is offset from the mean relation defined by astrometric systems. Follow-up observations of any eclipsing M-dwarfs found by MACHO, OGLE et al should be given the highest priority.

Third, how universal is $\Psi(M)$ ? Even without an absolute definition of $\Psi(M)$, it is apparent. particularly from open cluster work, that there are variations. The Pleiades appears to have $\Psi(M) \propto$ $M^{-1}$, matching results for local field stars (which data, as Henry and Becklin points out, may still be incomplete); Hillenbrand (1997), however, finds that the Orion core stars are closer to a Miller-Scalo function, peaking at $\sim 0.2 M_{\odot}$; and some young open clusters (eg IC 2391) seem to lack any latetype M-dwarfs. Are these differences real, or selection effects? Note that, unlike field-star surveys, selection effects in cluster surveys predominantly lie in including field stars, and overestimating $\Psi(M)$.

Fourth, are there significant differences between $\Psi(M)$ for single stars (and primaries) and companions? Imaging surveys for companions to white dwarfs (Becklin \& Zuckerman) and Mdwarfs (Henry, Oppenheimer), and radial velocity surveys of nearby stars all detect many M-dwarf companions, but few brown dwarfs - contradicting one's expectations given a mass function rising 
towards lower masses. Does this reflect significant differences in the physics of formation, or are selection effects still sufficient to account for this result?

Clearly, many other questions remain, notably whether brown dwarfs (or white dwarfs) have anything to do with MACHOs. These are issues for the next three years - or more.

\section{References}

D'Antona, F. \& Mazzitelli, I. 1983, Astr. Ap. 127, 149-152

Delfosse, X. et al, 1997, Astr. Ap., in press

Hillenbrand, L.A., 1997, AJ 113, 1733-1754

Nakajima et al, 1995, Nature 378,463-465

Rebolo, R. et al, 1996, ApJ 469, L53-56

Reid, I.N 1993, Science with astronomical near-IR surveys, ed. N Epchstein

Tsuji, T. 1964, Ann. Tokyo Obs. Ser. II, 9, 1-24 